\title{
Engineered nonlinear lattices
}

Clausen, Carl A. Balslev; Christiansen, Peter Leth; Torner, L.; Gaididei, Yuri Borisovich

Published in:

Physical Review E. Statistical, Nonlinear, and Soft Matter Physics

Link to article, DOI:

10.1103/PhysRevE.60.R5064

Publication date:

1999

Document Version

Publisher's PDF, also known as Version of record

Link back to DTU Orbit

Citation (APA):

Clausen, C. A. B., Christiansen, P. L., Torner, L., \& Gaididei, Y. B. (1999). Engineered nonlinear lattices. Physical Review E. Statistical, Nonlinear, and Soft Matter Physics, 60(5), R5064-R5067. https://doi.org/10.1103/PhysRevE.60.R5064

\section{General rights}

Copyright and moral rights for the publications made accessible in the public portal are retained by the authors and/or other copyright owners and it is a condition of accessing publications that users recognise and abide by the legal requirements associated with these rights.

- Users may download and print one copy of any publication from the public portal for the purpose of private study or research.

- You may not further distribute the material or use it for any profit-making activity or commercial gain

- You may freely distribute the URL identifying the publication in the public portal

If you believe that this document breaches copyright please contact us providing details, and we will remove access to the work immediately and investigate your claim. 


\title{
Engineered nonlinear lattices
}

\author{
C. Balslev Clausen and P. L. Christiansen \\ Department of Mathematical Modelling, Technical University of Denmark, DK-2800 Lyngby, Denmark \\ L. Torner \\ Laboratory of Photonics, Department of Signal Theory and Communications, Universitat Politecnica de Catalunya, \\ Barcelona, ES 08034, Spain \\ Yu. B. Gaididei \\ Bogulyobov Institute for Theoretical Physics, 252143 Kiev, Ukraine
}

(Received 28 May 1999)

\begin{abstract}
We show that with the quasi-phase-matching technique it is possible to fabricate stripes of nonlinearity that trap and guide light like waveguides. We investigate an array of such stripes and find that when the stripes are sufficiently narrow, the beam dynamics is governed by a quadratic nonlinear discrete equation. The proposed structure therefore provides an experimental setting for exploring discrete effects in a controlled manner. In particular, we show propagation of breathers that are eventually trapped by discreteness. When the stripes are wide the beams evolve in a structure we term a quasilattice, which interpolates between a lattice system and a continuous system. [S1063-651X(99)51311-1]
\end{abstract}

PACS number(s): 42.65.Wi, 42.65.Ky, 42.65.Tg

Nonlinear wave-mixing in a homogeneous nonlinear medium requires that the phase mismatch between the interacting waves be very small. Alternatively, the intrinsic phase mismatch can be compensated for in an inhomogeneous medium with a periodic grating. The latter method is known as the quasi-phase-matching (QPM) technique, which in the last few years has become widely employed in wave-mixing experiments due to the remarkable progress made in domaininversion techniques, notably for samples made of lithium niobate [1].

The potential of QPM extends far beyond the efficient phase matching of a single wave-mixing process which takes place in a sample with a regular, periodic grating. More complicated grating patterns can be fabricated, which results in media with unusual properties that can thus be engineered in a controllable fashion. This has been explored in a variety of devices that are based on creative grating designs. For example, temporal pulse compression [2], spatial beam compression with solitons [3], broadband phase matching [4], and even a quasiperiodic spectrum of wavelength conversion [5] has been demonstrated to occur in samples where the grating periodicity was broken in the longitudinal direction. Samples with grating patterns in the transverse direction have been used for beam tailoring [6], a wavelength converter with broad tuning range [7], and beam steering with solitons [8].

In this Rapid Communication we explore the possibility of producing transversely patterned gratings and consider an array of narrow grating stripes, as indicated in Fig. 1. Each stripe is composed of gratings with a narrow width, in the sense that the gratings are confined in the transverse direction to a width which is less than the beam width. Experimental setups usually require tightly focused beams with widths of $10 \sim 20 \mu \mathrm{m}$. Grating stripes with these widths can be implemented with the current state of the art lithographic QPM technology [6,9], but even smaller grating widths might be more challenging. So far, there have been no attempts to fabricate such narrow gratings, so the feasibility is an open question and only an experimental trial can give the answer. In practice, it can be anticipated that inhomogeneities appear along the interfaces between grating stripes and grating-free regions [10].

Our aim is to make a construction with a latticelike nature, in which each grating stripe plays the role of a site in the lattice. If the lattice features prevail, the beam evolution should essentially be governed by a discrete system, in close analogy to the beam evolution in an array of weakly coupled quadratic nonlinear waveguides [11]. In the present study we will restrict ourselves to the simplest possible grating design with discrete features. However, it should be remarked that more involved layouts can be envisaged with possible unique properties. For example, different lattices that each quasiphase match a particular wavelength may be interleaved to form a complex multi-wavelength discrete system. Inclusion of chirped, tilted, or dislocated gratings [8] in the region between the lattice sites could provide novel classes of nonlinear couplings between lattice sites. Furthermore, a lattice with competing nonlinearities may be constructed by exploiting the Kerr-like effects, which are inherent in the QPM technique [12].

In order to substantiate the investigation we consider a fundamental field at wavelength $\lambda_{1}$ and its second harmonic

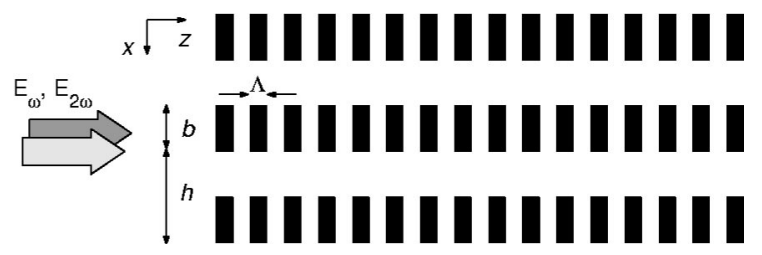

FIG. 1. Schematic top view of grating design of QPM structure. 
at wavelength $\lambda_{2}$ propagating in the structure, which is schematically shown in Fig. 1. In the slowly varying envelope approximation the dynamics of the envelope amplitudes of the fundamental, $a_{1}$, and the second harmonic, $a_{2}$, are governed by the equations

$$
\begin{gathered}
i \frac{\partial a_{1}}{\partial z}+\frac{1}{2} \frac{\partial^{2} a_{1}}{\partial x^{2}}+d(z, x) a_{1}^{*} a_{2} e^{-i \beta z}=0, \\
i \frac{\partial a_{2}}{\partial z}+\frac{1}{4} \frac{\partial^{2} a_{2}}{\partial x^{2}}+d(z, x) a_{1}^{2} e^{i \beta z}=0,
\end{gathered}
$$

where $\beta=\Delta k k_{1} \eta^{2}$ with $\Delta k=2 k_{1}-k_{2}$, is the phase mismatch, $k_{1}$ and $k_{2}$ are the wave number at each wavelength, and $\eta$ is a normalization parameter, which we set equal to the beam width. The relationships between normalized $(z, x)$ and physical $(Z, X)$ distances are given by $x=X / \eta$ for the transverse direction and $z=Z /\left(2 l_{d}\right)$ for the longitudinal direction, where $l_{d}$ is the diffraction length of the fundamental beam $l_{d}=\eta^{2} k_{1} / 2$. In lithium niobate with $\lambda_{1} \sim 1 \mu \mathrm{m}$ and $\eta \sim 15 \mu \mathrm{m}$ one finds $l_{d} \sim 1 \mathrm{~mm}$. The grating function from Fig. 1 is described by $d(z, x)$,

$$
d(z, x)=\sum_{n} g\left(x-x_{n}\right) \sum_{m} d_{m} e^{i \kappa m z},
$$

with $x_{n}=n h$, where $h$ is the distance between adjacent gratings. The grating wave number $\kappa=\pi / \Lambda$, where $\Lambda$ is the domain length, while $d_{m}$ are the Fourier coefficients for the grating, $d_{2 m}=0$ and $d_{2 m+1}=-2 i /(2 m+1)$. The comblike transverse grating structure is composed of a sequence of "'hat functions,' $g\left(x-x_{n}\right)$, which are each centered on $x_{n}$,

$$
g(x)=\mu(x-b / 2)-\mu(x+b / 2),
$$

where $\mu(x)$ is Heaviside's function and $b$ is the width of the gratings.

In the longitudinal direction, the coherence length $l_{c}=\pi /|\Delta k|$, is much shorter than any other scale. Therefore, we may average Eqs. (1) over a period of $l_{c}$. We assume first order QPM, i.e., $\Lambda \sim l_{c}$, and introduce $A_{1}=b \pi / 2\left\langle a_{1}\right\rangle$, $A_{2}=-i b \pi / 2\left\langle a_{2}\right\rangle \exp (-i \widetilde{\beta} z)$ for the scaled, averaged envelopes. In the lowest order approximation we find $[12,15]$

$$
\begin{gathered}
i \frac{\partial A_{1}}{\partial z}+\frac{1}{2} \frac{\partial^{2} A_{1}}{\partial x^{2}}+\frac{1}{b} \sum_{n} g\left(x-x_{n}\right) A_{1}^{*} A_{2}=0, \\
i \frac{\partial A_{2}}{\partial z}+\frac{1}{4} \frac{\partial^{2} A_{2}}{\partial x^{2}}-\widetilde{\beta} A_{2}+\frac{1}{b} \sum_{n} g\left(x-x_{n}\right) A_{1}^{2}=0,
\end{gathered}
$$

where $\widetilde{\beta}=\beta-\kappa$ is the residual phase mismatch. Equations (4) conserve both the power $I=\int_{-\infty}^{\infty}\left|A_{1}\right|^{2}+\left|A_{2}\right|^{2} \mathrm{~d} x$ and the Hamiltonian,

$$
\begin{aligned}
H= & \frac{1}{2} \int_{-\infty}^{\infty}\left(\left|\frac{\partial A_{1}}{\partial x}\right|^{2}+\frac{1}{4}\left|\frac{\partial A_{2}}{\partial x}\right|^{2}+\widetilde{\beta}\left|A_{2}\right|^{2}\right. \\
& \left.-\sum_{n} g\left(x-x_{n}\right)\left(A_{1}^{2} A_{2}^{*}+A_{1}^{* 2} A_{2}\right)\right) d x .
\end{aligned}
$$

As $b \rightarrow 0$ the stripes degenerate into a lattice of nonlinear impurities embedded in a linear medium. A fundamental prerequisite for the proper functioning of the lattice is that light is actually trapped at each individual site, similarly to the way light is trapped in modes of a linear waveguide. The present structure does not provide a refractive index change, so light should be trapped entirely by the presence of a grating stripe, which defines a narrow peak where nonlinear effects are present. It can be anticipated that at least with very narrow gratings such trapping will occur, since it has been shown that various types of the so-called impurity modes exist in quadratic nonlinear media with a single, isolated impurity embedded $[13,14]$. For the present structure, the relevant impurity modes are found by looking for the bound states of Eqs. (4) in the limit $b \rightarrow 0, h \rightarrow \infty$. Since this is a linear medium with a single, isolated nonlinear impurity, the modes are readily found analytically. This suggests that with narrow gratings, light may indeed be trapped at each site. However, we should keep in mind that the limit of vanishing $b$ makes the whole analysis invalid. The gratings will in that case disappear and the transformation $a_{j} \rightarrow A_{j}$ will be singular. Still, with $b \rightarrow 0$, Eqs. (4) will be relevant as a model of photonic crystals $[14,16]$. In the general case with $b \neq 0$ we have to resort to numerical calculations. These demonstrate that modes which are exponentially localized in the linear regions exist with any site width. We therefore expect that the beam dynamics also in that case will possess discrete features.

In order to proceed with the analysis, we follow a path similar to a treatment of impurities in the nonlinear Schrödinger equation [17]: We solve Eqs. (4) in each linear layer and express the solution in terms of the amplitudes at the nonlinear sites. In the limit $b \rightarrow 0$, the solutions in the linear layers can be connected by integration across the nonlinear sites yielding that the evolution of these site amplitudes is given by a set of coupled ordinary differential equations,

$$
\begin{gathered}
i \frac{\mathrm{d} B_{n}}{\mathrm{~d} z}+\frac{1}{2 h^{2}}\left(B_{n+1}+B_{n-1}-2 B_{n}\right)+\frac{1}{h} B_{n}^{*} D_{n}=0, \\
i \frac{\mathrm{d} D_{n}}{\mathrm{~d} z}+\frac{1}{4 h^{2}}\left(D_{n+1}+D_{n-1}-2 D_{n}\right)-\widetilde{\beta} D_{n}+\frac{1}{h} B_{n}^{2}=0,
\end{gathered}
$$

$B_{n}$ and $D_{n}$ are the amplitudes on the $n$th site of the fundamental and second harmonic, respectively. In deriving Eqs. (6) we assumed the following ordering for $B_{n}$ :

$$
\frac{\partial}{\partial z} \sim \epsilon, \quad B_{n} \sim \epsilon, \quad B_{n+1}+B_{n-1}-2 B_{n} \sim \epsilon B_{n},
$$

and a similar ordering for $D_{n}$. The parameter $\epsilon$ indicates the relative magnitude of the terms. See Ref. [17] for an explicit derivation. The ordering suggests that the discrete model (6) will not be accurate with large beam amplitudes. Also, the derivation is based on the limit $b \rightarrow 0$, so narrow gratings are necessary for the accuracy of Eq. (6). However, we have observed from numerical simulations that when these conditions are not fulfilled, meaning that $b$ is nonvanishing or even large, or the beam amplitudes are large, the beam dynamics still bears the characteristic features of discreteness. But in that case it is not captured by the simple Eqs. (6). We expect that as $b$ is increased from zero, the QPM structure will 

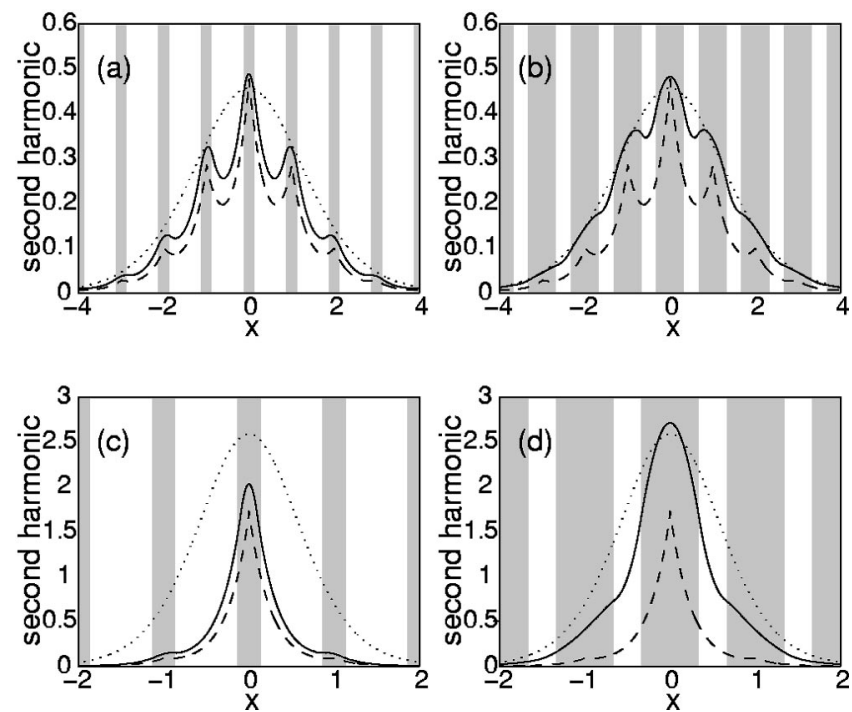

FIG. 2. Second harmonic part of stationary localized modes (solid lines) with $\widetilde{\beta}=1$ and $h=1$. Superimposed are profiles of continuous solitons with $b=h$ (dotted lines) and discrete solitons with $b=0$ (dashed lines). The shaded areas indicate the regions where $g(x)=1$, i.e., the nonlinear regions. (a) $b=0.3, q=0.25$, (b) $b$ $=0.7, q=0.25$, (c) $b=0.2, q=1.5$, and (d) $b=0.7, q=1.5$.

interpolate between a fully discrete system $(b=0)$ and a continuous medium $(b=h)$. Thus, we refer to the structure as a quasilattice.

With the purpose of exposing the interpolating character of the quasilattice, we have numerically found the localized, stationary modes of the structure, i.e., we have inserted $A_{1}$ $=A(x) \exp (i q z)$ and $A_{2}=B(x) \exp (i 2 q z)$ in Eqs. (4) and solved the resulting boundary value problem. We expect that at least for some parameter values such stationary solutions will exist and be stable, since in the two limits $b=0$ and $b$ $=h$ they will tend to the familiar discrete [11] and continuous solitons [18], respectively. In Fig. 2 we plot the amplitude $B(x)$ of the numerically found stationary modes with $h=1, \widetilde{\beta}=1$ and two different values of $b$ and $q$. The corresponding profiles of the solitons in the limits $b=0$ and $b$ $=h$ are superimposed on the plots as dashed and dotted lines, respectively. It should be clear that with small $b$ the stationary modes resemble the discrete soliton, while with large $b$ they resemble the continuous solitons. Also, we note that with large amplitudes ( $q$ large) a relatively small value of $b$ is required in order to obtain a good resemblance to the discrete solitons.

Although the appearance of the profiles of the stationary modes indicates in a neat way how the quasilattice interpolates between a discrete lattice and the continuum, it does not provide a complete characterization of the quasilattice. To this end, it will be necessary also to investigate the mobility of the localized modes. It is well known that discrete solitons lose energy if they are forced to move across the lattice, and that their mobility decreases as their power content is increased. In contrast, continuous solitons are in a system with translational symmetry and can move freely with any velocity (angle). For a review of the properties of moving breathers in the context of optics see, e.g., Ref. [19]. As a qualitative measure of the mobility of modes in the quasilattice we calculate the Peierls-Nabarro (PN) potential of the modes.
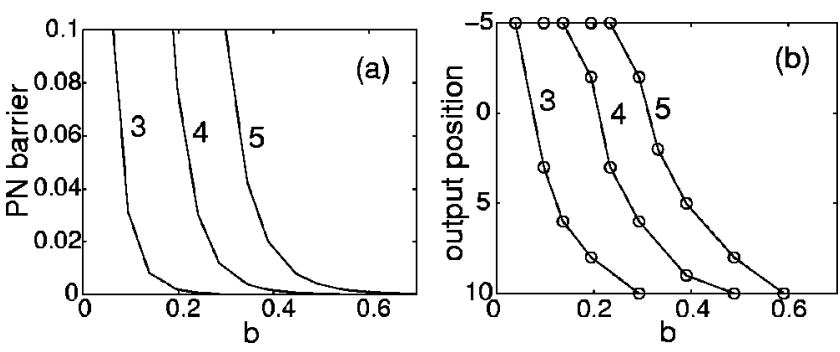

FIG. 3. (a) Peierls-Nabarro barrier and (b) output position in $x$ of peak amplitude at $z=30$ vs $b$. Input position and velocity are -5 and 0.5 , respectively. Numbers indicate value of power, $I . \tilde{\beta}=3$, $\beta=400$, and $h=1$.

The modes shown in Fig. 2 are called odd modes, since they have their peak amplitude centered on a site, thus yielding a profile with an odd number of peaks. The so-called even modes, with an even number of peaks which are centered in between two sites, can also be found. The PN potential is the difference in Hamiltonian energy $H$ between the even and the odd mode with a fixed power $I$. The idea behind using the PN potential as a measure of mobility relies on the assumption that when the odd mode moves from one site to the next it transforms into an even mode in the process. The difference in Hamiltonian energy is thus a potential barrier that must be overcome in the process of translation [20]. It seems plausible that an odd mode actually does not follow a path exactly via an even mode when moving from site to site, but probably the PN potential can still give a qualitative suggestion for the mobility. In Fig. 3(a) we plot the PN barrier versus $b$ for the modes in the quasilattice with $\widetilde{\beta}=3, h=1$, and three values of $I$.

Figure 3 also includes a measure of the actual mobility of the modes in the quasilattice. We have made a careful numerical study solving Eqs. (1) with a variety of initial conditions and parameter values. For the numerical simulations we chose parameter values that are relevant for tightly focused beams in a sample made of lithium niobate, yielding $\beta \sim 400$. We measure the mobility by launching a mode with an initial phase tilt, $\mathrm{v}: a_{j} \rightarrow a_{j} \exp (i j \mathrm{v} x)$, which will induce a velocity in the mode, forcing it to move across the lattice. The results of a series of such numerical experiments are indicated in Fig. 3(b). The imposed initial velocity is $\mathrm{v}$ $=0.5$ and the initial position is $x_{0}=-5$. We plot the output position in $x$ after the mode has propagated a distance $L$ $=30$ in $z$. Comparing the PN barrier and the actual mobility
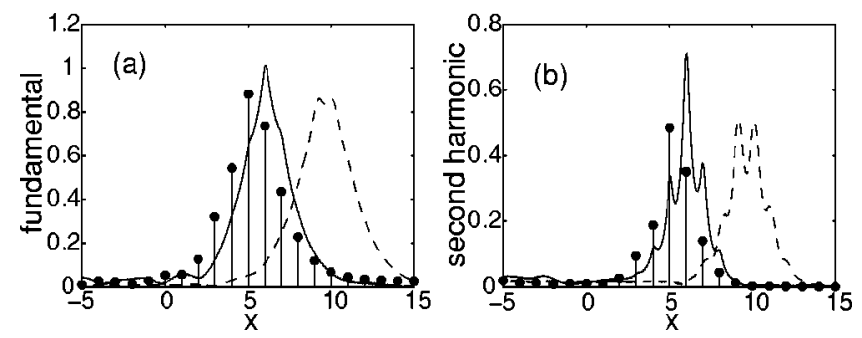

FIG. 4. Output at $z=30$. Stemplot (bullets on lines) are results from discrete Eqs. (6) and lines are from Eqs. (1) with $b=0.25$ (solid lines) and $b=0.5$ (dashed lines). $\widetilde{\beta}=1, \beta=400$, and $h=1$. Input position and velocity is $x_{0}=-10$ and $\mathrm{v}=0.5$, respectively. (a) Fundamental and (b) second harmonic. 
determined from numerical simulations, the qualitative agreement is visible. They both reveal that localized modes in the quasilattice interpolate between the tendency to be trapped in the discrete limit and the free translation in the continuous limit.

Finally, we give an example where the simple discrete system (6) predicts correctly the behavior of the beam dynamics in the original system (1). A mode with an initial phase tilt $\mathrm{v}=0.5$ is launched with an initial position $x_{0}=$ -10 , and the output position at $z=30$ is shown in Fig. 4. The results from the discrete system (6), which are marked with bullets, show that the mode has been slowed down by the discreteness and is positioned at $x=5$. The results from numerical simulations of Eqs. (1) with $b=0.25$ show that the mode moves in the quasilattice in agreement with Eq. (6), indicating that the quasilattice is effectively discrete. The results for the simulations with $b=0.5$ show that the mode moves in agreement with the translational symmetry of a continuous system, since the mode has not been slowed down. Still, features reminiscent of discreteness are visible in the appearance of the profiles of the freely moving mode.

In summary, we have investigated engineered quasiphase-matched samples with transverse comb patterns and found that beam evolution in these samples possesses the characteristic properties of a discrete system. By means of investigation of the breather profiles and breather mobility, we have shown that the samples interpolate between fully discrete systems and continuous systems, the tuning parameter being the width $b$ of each grating line. The quasilattices make it possible to devise discrete quadratic lattices with properties that can be engineered to meet specific needs.

We are most grateful to M. M. Fejer for important discussions and remarks.
[1] M.M. Fejer, in Beam Shaping and Control with Nonlinear Optics, edited by F. Kajzar and R. Reinisch (Plenum, New York, 1998), pp. 375-406; F. Laurell, Opt. Mater. 11, 235 (1999).

[2] M.A. Arbore, O. Marco, and M.M. Fejer, Opt. Lett. 22, 865 (1997); M.A. Arbore, A. Galvanauskas, D. Harter, M.H. Chou, and M.M. Fejer, ibid. 22, 1341 (1997); G. Imeshev, A. Galvanauskas, D. Harter, M.A. Arbore, M. Proctor, and M.M. Fejer, ibid. 23, 864 (1998).

[3] L. Torner, C. Balslev Clausen, and M.M. Fejer, Opt. Lett. 23, 903 (1998).

[4] K. Mizuuchi and K. Yamamoto, Opt. Lett. 23, 1880 (1998).

[5] S. Zhu et al., Phys. Rev. Lett. 78, 2752 (1997); Science 278, 843 (1997).

[6] G. Imeshev, M. Proctor, and M.M. Fejer, Opt. Lett. 23, 673 (1998).

[7] P.E. Powers, T.J. Kulp, and S.E. Bisson, Opt. Lett. 23, 159 (1998).

[8] C. Balslev Clausen and L. Torner, Phys. Rev. Lett. 81, 790 (1998); Opt. Lett. 24, 7 (1999).

[9] L.E. Myers, R.C. Eckardt, M.M. Fejer, R.L. Byer, and W.R. Bosenberg, Opt. Lett. 21, 591 (1996); B. Widiyatmoko, K. Imai, M. Kourogi, and M. Ohtsu, ibid. 24, 315 (1999).

[10] M.J. Missey, V. Dominic, L.E. Myers, and R.C. Eckardt, Opt.
Lett. 23, 664 (1998); Y. Tomita, M. Sugimoto, and K. Eda, Appl. Phys. Lett. 66, 1484 (1995); K. Eda, M. Sugimoto, and Y. Tomita, ibid. 66, 827 (1995).

[11] T. Peschel, U. Peschel, and F. Lederer, Phys. Rev. E 57, 1127 (1998).

[12] C. Balslev Clausen, O. Bang, and Yu.S. Kivshar, Phys. Rev. Lett. 78, 4749 (1997).

[13] C. Balslev Clausen, J.P. Torres, and L. Torner, Phys. Lett. A 249, 455 (1998).

[14] A.A. Sukhorukov, Yu.S. Kivshar, and O. Bang, Phys. Rev. E 60, R41 (1999).

[15] L. Torner and G.I. Stegeman, J. Opt. Soc. Am. B 14, 3127 (1997).

[16] V. Berger, Phys. Rev. Lett. 81, 4136 (1998).

[17] Yu.B. Gaididei, P.L. Christiansen, K.Ø. Rasmussen, and M. Johansson, Phys. Rev. B 55, R13 365 (1997).

[18] A.V. Buryak and Yu.S. Kivshar, Phys. Lett. A 197, 407 (1995); L. Torner, Opt. Commun. 114, 136 (1995).

[19] A.B. Aceves, C. DeAngelis, T. Peschel, R. Muschall, F. Lederer, S. Trillo, and S. Wabnitz, Phys. Rev. E 53, 1172 (1996).

[20] M. Peyrard and M.D. Kruskal, Physica D 14, 88 (1984); Yu.S. Kivshar and D.K. Campbell, Phys. Rev. E 48, 3077 (1997). 